\title{
Impact of Observatory Visitor Centres on the Public's Understanding of Astronomy
}

\author{
Kimberly Burtnyk \\ Manager of Evaluations, \\ California Science Centre, \\ 700 State Drive, \\ Los Angeles, CA 90037, USA
}

\author{
Received 1999 October 15, accepted 2000 September 4
}

\begin{abstract}
In recent years, formal astronomy education has become an issue of great interest. Indeed, some boards of education now officially include astronomy in their curricula. While formal astronomy education continues to gain attention, informal astronomy education for the general public has rarely been addressed. One valuable source of informal astronomy education is the observatory 'visitor centre' Observatories draw thousands of visitors each year, and as such represent a golden opportunity for the astronomical community to communicate directly with the public. This paper summarises an exploratory study of the cognitive and affective impacts of a visit to the visitor centres located at Mount Stromlo and Siding Spring Observatories.
\end{abstract}

Keywords: astronomy education—observatory visitor centres—informal education

\section{Introduction}

Throughout history, astronomy has connected people together, spiritually, scientifically, and even literally, in the realisation that we are all stardust. In this regard, astronomy is truly a science for all people. The power of astronomy lies in its aesthetic appeal, but the continuing loss of the night to the lights of civilisation means that people today are exposed less and less to a beautiful star-filled sky. Yet, while the stars are disappearing, the keen interest in all things astronomical, among many people, is not. Subsequently, there are now myriad ways for people wishing to learn more about astronomy to access information from books, magazines, specialty television programs, planetaria and the internet.

These are all valuable resources for astronomy education for a wide audience. Yet while they play a role in educating the public they are not the only such sources of astronomy for the interested individual. In fact, one resource for astronomy information and education that has rarely received formal research attention are the visitor interpretive centres located on-site at many of the world's observatories. Two such visitor centres associated with Australian observatories are the 'Exploratory' exhibitions at Mount Stromlo Observatory (MSO) in Canberra, and at Siding Spring Observatory (SSO) near Coonabarabran, New South Wales.

\section{The Setting}

Siding Spring Observatory is located in scenic Warrumbungle National Park in northern New South Wales, Australia. It is blessed with some of the darkest and clearest skies anywhere, and astronomers from around the world use its eight telescopes. The most famous resident of the observatory is the Anglo-Australian Telescope (AAT). With a primary mirror nearly four metres in diameter it is the largest optical telescope in Australia.
Mount Stromlo Observatory is one of Australia's oldest observatories. Nestled in the Brindabella Range on the outskirts of Canberra, it is home to five telescopes, the largest of which has a modest 1.9 metre diameter primary mirror-Australia's third largest telescope.

The visitor centres at both observatories are located in the immediate vicinity of the largest telescopes on site. Visitors can view these telescopes from 'galleries' located in each of the domes. Mount Stromlo also conducts regularly scheduled slide shows and guided tours of two telescopes on site, including the $1.9 \mathrm{~m}$.

An important difference between the two visitor centres is their respective ages: Siding Spring's is 25 years old, and Mount Stromlo's is just three years old. The content and design of the exhibitions therefore reflects the eras in which they were constructed. Siding Spring boasts a primarily text based (museum like) exhibition that was common at the time of its construction; Mount Stromlo boasts new and highly interactive exhibits, common in modern science centres.

\section{The Research Method}

This research took place between May 1998 and April 1999. The initial phase of the study took the form of on-site open-ended interviews where adult visitors to Mount Stromlo and Siding Spring observatories were randomly selected upon entry into the Exploratory exhibitions, unobtrusively observed throughout their visit, and interviewed upon exit. Adults chosen for interviews were alone or in pairs, and without young children. Childless respondents were chosen since the desired interview data required that visitors had not been influenced by the needs of children during their visit. In total 51 Siding Spring and 28 Mount Stromlo visitors were interviewed. Interview questions related to exhibits on which visitors spent some time, as well as questions 
relating to the entire visit experience. Cognitive and affective responses were sought.

The last phase of the study took the form of followup telephone interviews, which were conducted between six weeks and ten months after initial visit experiences. Follow-up interviews were used to reveal the robustness and longevity of visitor recollections, behaviour and/or attitude changes resulting from the visit, and cognitive and affective outcomes. In total, 39 follow-up interviews were conducted: 26 from Siding Spring, and 13 from Mount Stromlo, representing roughly half of all visitors interviewed at each observatory.

\section{Research Findings}

\subsection{Who visits Observatories and why?}

Visitors to both observatories possessed a wide range of interest and knowledge levels. Some considered their knowledge to be nearly non-existent, while others expressed a more serious interest, demonstrated by describing their use (recently or in the past) of field guides, binoculars, or even telescopes while looking at the night sky, and by expressing an interest in various sources of astronomical information. Perhaps surprisingly, no visitors were found to have any extensive formal astronomy educational training, nor would be described (nor did they describe themselves) as 'amateur astronomers'.

Most visitors were tourists, and in the case of Siding Spring, were taking a break on a much longer driving journey. No visitors were in the area deliberately and exclusively to see SSO and no visitor was found to be a resident of any of the communities immediately surrounding the observatory. This is not surprising given the remote location of the observatory, and the low population density in surrounding areas. Accordingly, the situation was quite different for Mount Stromlo, where half of all interviews involved at least one resident of Canberra who was escorting visiting relatives. The other half of MSO's visitors was either local or out of town tourists who (unlike SSO visitors) had deliberately set out to visit the observatory on that particular day.

At both observatories, the most commonly quoted reason for stopping was curiosity; some visitors were curious because they had heard of Mount Stromlo or Siding Spring and wanted to see it, while others were curious because they had not heard of the observatory and wanted to know what it was. Some Siding Spring visitors saw the dome of the AAT from afar and decided to take a closer look. Others dropped in only after seeing a sign for the observatory upon their arrival in Coonabarabran. In fact, some had never heard of Siding Spring until they arrived in town. The case was similar for Mount Stromlo where roughly one in three visitors had not heard of the observatory before they decided to visit.
Once the decision had been made to visit the observatory more than half of Mount Stromlo and Siding Spring visitors expressed that their motivation for visiting the site was to see a telescope. Furthermore, many visitors at both locations expressed a desire to look through a telescope, as the following examples illustrate [in the excerpts, $\mathrm{M} / \mathrm{F}$ denotes the gender of the respondent, I denotes 'interviewer', R denotes 'respondent', and the number denotes the respondent's age group]:

\section{F 30s [MSO] \\ I would have liked to have seen, been able to look through, even the one that was up here, it would have been nice to have actually been able to look and see out into space, I was looking forward to that. \\ $\mathrm{M}$ 60s [SSO] \\ I'd like to look through a telescope like this one!}

It deserves mentioning that Mount Stromlo conducts a public observing program and Siding Spring does not. It is also important to note that the Stromlo Exploratory houses a 14 inch telescope that is to be used when planets and the moon are visible in daytime skies. (This is the instrument being referred to by the first visitor above.) However, over the course of this study, that instrument was never seen to be in use.

\subsection{Results of Follow-up Interviews}

Following the example of museum researchers (Falk \& Dierking 1992), the first question on the follow-up questionnaire was simply: 'Tell me everything you remember from your visit'. This approach encourages unprompted, unbiased, open-ended responses involving both cognitive and affective recollections. Visitors were also asked to recall which memories were clearest in their minds, specific exhibits, and whether or not they 'learned' anything new.

Responses to these questions revealed surprising diversity. Besides specific exhibits, visitors also recalled a wide variety of miscellaneous memories from their visit (see Table 1). Affective responses like 'we had a great time', 'we had an enjoyable day', weather conditions, or events not directly related to the observatory visit itself, were also frequently part of the 'memories' recalled by visitors.

Not surprisingly, the most oft-quoted memories from both locations were the telescopes. When then asked which memories were clearest in their minds, the telescopes, in both locations, were also mentioned the most.

Over the course of the entire follow-up interview, visitors frequently recalled specific exhibits. Table 2 reveals that fourteen SSO exhibits and ten MSO exhibits were mentioned. Of those SSO exhibits mentioned, seven were text-based displays, and seven were handson or interactive exhibits. Of the MSO exhibits mentioned, six were hands-on or interactive in nature.

Of all responses, three exhibits were recalled the most. At Mount Stromlo, the two most commonly 
Table 1. Visitor recollections

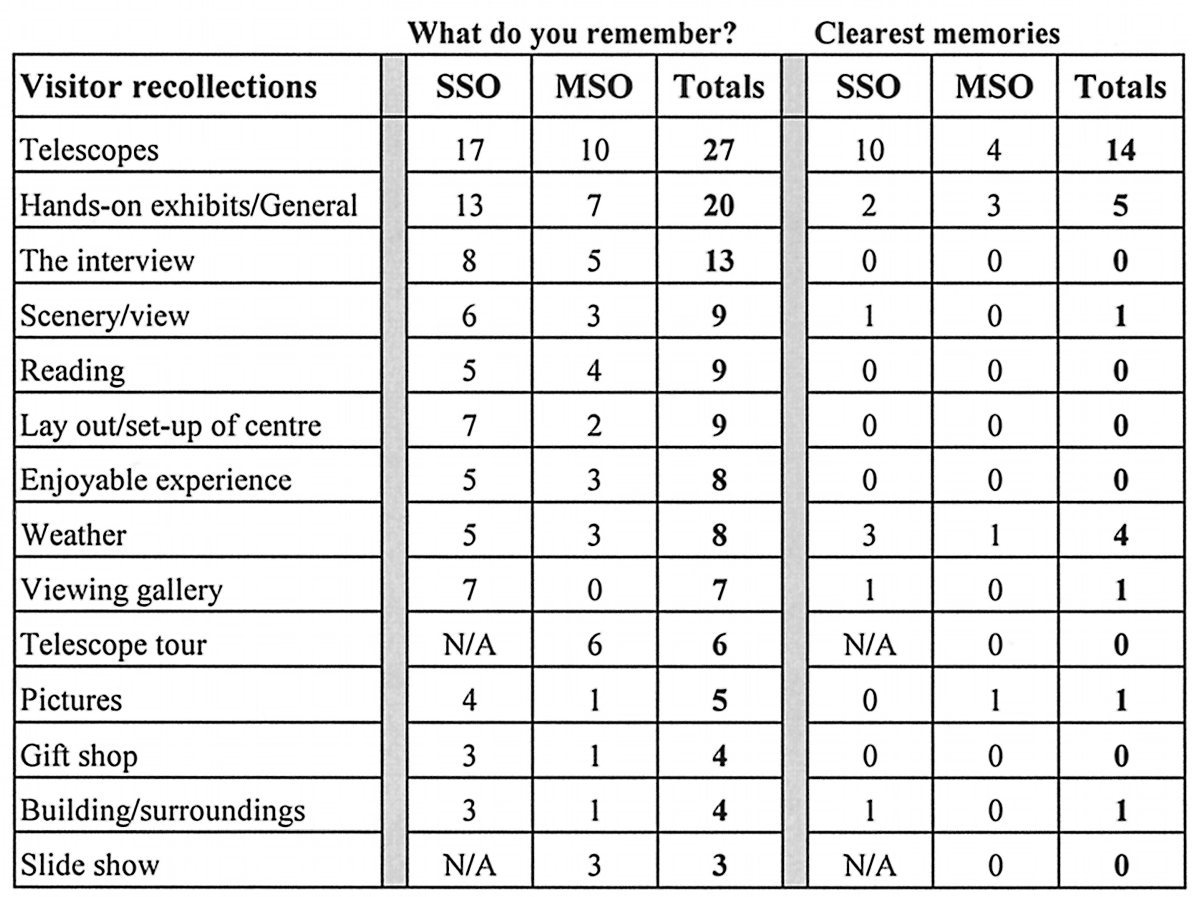

Table 2. Specific exhibit recollections

\begin{tabular}{|c|c|c|c|c|c|c|}
\hline \multicolumn{4}{|c|}{ Specific exhibits } & \multicolumn{3}{|c|}{ Clearest memories } \\
\hline Exhibit recollection & SSO & MSO & Totals & SSO & MSO & Totals \\
\hline Red/Blue Shift+ & 1 & N/A & 1 & 0 & N/A & 0 \\
\hline Dist. to Stars / Star Rulers*+ & 1 & 4 & 5 & 0 & 0 & 0 \\
\hline Composite mirrort & 2 & N/A & 2 & 0 & $\mathrm{~N} / \mathrm{A}$ & 0 \\
\hline Coke cans / Milk cartons ${ }^{*}+$ & 5 & 4 & 9 & 1 & 2 & 3 \\
\hline Light bends around corners+ & 3 & N/A & 3 & 0 & N/A & 0 \\
\hline Computers*+ & 6 & 1 & 7 & 1 & 0 & 1 \\
\hline Solar System model & 2 & N/A & 2 & 1 & N/A & 1 \\
\hline Quasars & 1 & N/A & 1 & 0 & N/A & 0 \\
\hline Orrery & 1 & N/A & 1 & 1 & N/A & 1 \\
\hline Aztec calendar & 1 & N/A & 1 & 0 & N/A & 0 \\
\hline Meteorites*+ & 3 & 5 & 8 & 2 & 2 & 4 \\
\hline 14 inch telescope & N/A & 2 & 2 & N/A & 0 & 0 \\
\hline Design a Solar System+ & N/A & 3 & 3 & N/A & 0 & 0 \\
\hline Heliostat+ & N/A & 1 & 1 & N/A & 0 & 0 \\
\hline Trekking through the Universe+ & N/A & 2 & 2 & N/A & 0 & 0 \\
\hline Ultra-violet+ & N/A & 1 & 1 & 0 & 0 & 0 \\
\hline Schmidt plates ${ }^{*}$ & 1 & 0 & 1 & 1 & 0 & 1 \\
\hline Scale of the Universe & 1 & N/A & 1 & 1 & N/A & 1 \\
\hline Hertzsprung-Russel diagram & 1 & N/A & 1 & 0 & N/A & 0 \\
\hline Seismograph+ & N/A & 1 & 1 & N/A & 0 & 0 \\
\hline Total no. of exhibits recalled & 14 & 10 & & 7 & 2 & \\
\hline
\end{tabular}

* Indicates exhibits or concepts common to both Exploratories.

+ Indicates exhibits which are hands-on or interactive in nature. 
recalled exhibits were the Meteorites and the 'Milk Cartons'. At Siding Spring the most common exhibits recalled were the Computer terminals, and 'Coke Cans'. ('Coke Cans' and 'Milk Cartons' use familiar objects to demonstrate the difference in gravity between the planets in the Solar System.) A close third at Siding Spring was also the Meteorites exhibit.

Looking at Table 2 it should also be noted that Mount Stromlo's Star Rulers exhibit was also recalled numerous times. However, the context of the recollection was significantly different from all other exhibit memories. In fact, in each of the four Star Rulers recollections visitors recalled only that they were not able to successfully work the exhibit:

F30s

I: Do you recall any specific exhibits?

R: [Star Rulers]. I couldn't do it. It was difficult to follow.

M60s

I: Do you recall any specific exhibits?

$\mathrm{R}$ : There was one machine where you could line up the stars if you were clever enough, but I couldn't do it. I had a go but I couldn't do it.

This is an important finding that reveals the failure of this exhibit to communicate effectively with those who chose to use it (i.e. the general public). More importantly, this example dramatically illustrates the importance of understanding visitor demographics when designing exhibits.

\subsection{Cognitive Impact of a Visit to an Observatory}

While the point of the first question was to reveal basic visitor recollections, other follow-up questions sought to reveal specific cognitive and affective impacts of the visit. Questions like, is there anything you remember from your visit that you did not know before your visit, what was the effect of the visitor centre on your visit, and overall, what if anything did you gain from your visit, were asked to reveal cognitive impact.

There were three main groups of cognitive responses (Table 3). Visitors either indicated that they did 'learn' something new and could specify what that was, or they felt they learned something but could not specify, or they stated that they increased their general knowledge, but again, could not specify.

Seven respondents recalled specific details of new information that they 'learned' during their visit. Topics they mentioned included light pollution, different types of telescopes, quasars, and international collaboration. Transcripts of two such cases are found below:

F 30s [SSO]

I: What effect did the visitor centre have on your overall visit to the site?

R: It explained everything why the observatory was there, Canberra was shedding too much light, which is why this was set up.

I: Was that something you knew before?

R: No.

\section{60s [SSO]}

[There was] an exhibit explaining something about Quasars, where they were found, it said age was 15 billion years which I found to be absolutely incredible because I thought that was the age of the universe, there was an exhibit about the quasars.

While these visitors could specify information that they 'learned' during their visit, other visitors could not specify, though they genuinely felt that they learned something. For example:

F 50s [SSO]

I: Can you recall anything that was new to you?

R: Heaps just can't think of them at the moment

I: What were you thinking or feeling as you were walking around?

$\mathrm{R}$ : That it was a learning experience

Whether or not this response is an honest one (that is, the visitor could recall details at a later date) is impossible to know.

The most common response to probing recollections of new information obtained during the visit to the observatory was some reference to 'general knowledge'. Other references were made that implied general knowledge without the visitor actually utilising those exact words. Examples are found below.

\section{40s [MSO]}

It confirmed previous knowledge by actually being there and touching it [meteorite], being around it, it reinforced previous information that I had.

Table 3. Cognitive outcome

\begin{tabular}{|c|c|c|c|c|c|c|c|}
\hline Learning outcome & \multicolumn{2}{|c|}{ MSO } & \multicolumn{2}{|c|}{ SSO } & \multicolumn{2}{|c|}{ Totals } & \\
\hline General knowledge & $4 \mathrm{M}^{*}$ & $2 \mathrm{~F}$ & $4 \mathrm{M}$ & $3 \mathrm{~F}$ & $8 \mathrm{M}$ & $5 \mathrm{~F}$ & 13 \\
\hline Specified & $1 \mathrm{M}$ & $2 \mathrm{~F}$ & $1 \mathrm{M}$ & $3 \mathrm{~F}$ & $2 \mathrm{M}$ & $5 \mathrm{~F}$ & 7 \\
\hline Yes, but can't recall & $1 \mathrm{M}$ & $0 \mathrm{~F}$ & $2 \mathrm{M}$ & $4 \mathrm{~F}$ & $3 \mathrm{M}$ & $4 \mathrm{~F}$ & 7 \\
\hline NO & $4 \mathrm{M}$ & $1 \mathrm{~F}$ & $4 \mathrm{M}$ & $2 \mathrm{~F}$ & $6 \mathrm{M}$ & $3 \mathrm{~F}$ & 9 \\
\hline 'Yes' total & $6 \mathrm{M}$ & $4 \mathrm{~F}$ & $7 \mathrm{M}$ & $10 \mathrm{~F}$ & $13 M$ & $14 \mathrm{~F}$ & \\
\hline
\end{tabular}

${ }^{*} \mathrm{M}-$ Male respondents; $\mathrm{F}-$ Female respondents 
F 30s [SSO]

I: What did you gain from your visit

R: Well you always broaden your knowledge when you go to places like that. It's putting just something tangible into what you've read about the place...

Interestingly, 27 out of 39 follow-up respondents themselves perceived their visit as a learning experience.

\subsection{Affective Impact of a Visit to an Observatory}

In this context, 'affective impact' refers to those thoughts, feelings, or memories raised by visitors, that were not simply factual in nature. They were experiential rather than concrete, they were subjective and varied, and they were highly personal in nature, as opposed to cognitive memories where visitors could recall specific factual details of things they saw at the observatory. Despite the richness of affective data, the task of classifying and measuring affect has not been taken up by many researchers, perhaps because of the inherent difficulties in doing so (Rennie \& McClafferty 1996).

There is no doubt that astronomy is highly affective in nature; most of what the general public knows and enjoys about astronomy stems from photographs taken by the world's observatories. Therefore, it was deemed important early in this study to attempt to measure the affective impact of the visit to an observatory.

The follow-up phase of the study proved to be a valuable measure of the lasting impact of affect. The questions: was there anything new that you remember, what did you gain from your visit, and what were you thinking and feeling when you were walking around, all revealed an interesting profile of the affective impact of a visit to an observatory. Overall, 35 of 39 respondents recalled memories that were particularly affective in nature. These memories ranged from recalling aspects of the physical environment, to pondering fundamental philosophical questions about the scale and nature of the universe.
F 50s [MSO]

I: What do you remember?

R: It was cold, I remember the telescope, and wandering around, I remember being entirely fascinated by the size of the universe, and our interview

I: What were you thinking or feeling when you were on site?

$\mathrm{R}$ : That the universe is fascinatingly awesome, I was in awe of it all.

Other visitors mentioned the beauty of astronomy, and even the relationship between the universe and religion. In one instance, a female Mount Stromlo visitor in her 40 s expressed her interest in the aesthetic appeal of astronomy. She said:

I remember those photos because I know David Malin, so I'm interested in those photographs. I've seen them before, and I think they're beautiful, to me they're like beautiful crystals.

In another case, a male Mount Stromlo visitor in his 50s made a remark of a particularly affective flavour, questioning the nature of existence itself:

I think all of us that have looked at the stars have thought what's that all about? What are we all about? Are these two linked? Did we come from there? As I said, the fundamental questions .... Every society has had the belief that somehow or other the fundamental questions, the answers to them lies up there which has led people to develop myths and legends some of which translated into practical developments, ... fundamental questions, what's it all about?

Other affective memories were the less tangible or describable things, such as visitors recalling a 'sense' of the excitement of having the unique experience of being at the observatory. The most common affective response was 'we had an enjoyable experience'.

\subsection{Behavioural/Attitudinal Impact of a Visit to an Observatory}

The notion of 'impact' is not simply defined by the things people remember or feel. Impact is also defined by the behavioural or attitudinal changes that may or

Table 4. Attitude or behavioural impact

\begin{tabular}{|c|c|c|}
\hline SSO & Yes & No \\
\hline Purchased astronomy related books since visit & 1 & 14 \\
\hline Pay more attention to astronomy in the media since visit & 6 & 14 \\
\hline Look at the stars more since visit & 3 & 11 \\
\hline Changed opinion of astronomy research since visit & 4 & 13 \\
\hline MSO & Yes & No \\
\hline Purchased astronomy related books since visit & 0 & 5 \\
\hline Pay more attention to astronomy in the media since visit & 2 & 10 \\
\hline Look at the stars more since visit & 1 & 5 \\
\hline Changed opinion of astronomy research since visit & 2 & 10 \\
\hline
\end{tabular}


may not have taken place after an encounter with some new information or a new experience (Perry 1993). To be thorough, the part of impact that involves behaviour and attitude was also investigated in this study. To this end, visitors were asked: As a result of your visit, do you pay more attention to astronomy in the media, did you purchase any astronomy books or magazines, how do you feel about astronomy research, and do you look at the stars more than you used to before your visit? Table 4 outlines the results of this line of investigation.

The numbers of reported changes were small, but they were non-zero. Furthermore, responses were all positive in tone and no visitor was found to have developed a negative attitude or opinion as a result of their visit. Mount Stromlo visitors reported slightly fewer incidents of attitude of behaviour changes than did SSO visitors, but since the sample size was small, generalisations cannot be made.

\section{Discussion and Conclusions}

This study, though necessarily limited in scope, revealed much about the experience of visiting an observatory and the role of visitor centres in public outreach. On-site interviews revealed that visitors were truly members of the general public who arrived expecting to see a telescope but not necessarily expecting the presence of the visitor centre. It is encouraging then, that visitors left the observatories believing that the Exploratory was important to their visit experience and that their visit would have been somehow 'less' without it. More importantly, visitors indicated that the location of the visitor centre next to the telescopes added the crucial elements of context and relevance to the visit. As one visitor to Mount Stromlo succinctly put it:

F50s: You can't have one without the other in a terribly meaningful way.

Follow-up interviews revealed that some visitors felt they had a learning experience, and some demonstrated attitude or behaviour changes resulting from their visit. It was also apparent that the affective impact of the visit was strong, and closely related to the location of the centre. This affective response is not trivial since some researchers believe that:

'the fundamental educational aim is the affective domain, and [Wellington] points out that by achieving this aim, science centres are more likely to make their indirect contribution to higher order cognitive learning' (Rennie \& McClafferty 1996).

The affective nature of astronomy may mean that this type of response is particularly important to invoke. Pretty pictures and beautiful scenery may indeed provide the impetus for further investigation, and greater appreciation of the science. These sorts of communication practices should form the basis of any outreach activities and should be encouraged.

Follow-up interviews also revealed that the textbased design of the 25 year-old Siding Spring exhibition is not any less effective than the three year-old Mount Stromlo Exploratory, which is outfitted with new interactive exhibits. (Adult visitors in particular seemed to relate better to text based exhibits.) In both cases, the exhibits that were recalled the most, and the most fondly, were those which adhere to the basic rules of good exhibit design (Burtnyk 2000, p.33): they are simple, relevant, concrete, unique, and visitors have total control over the interaction. Also, they are experiential rather than conceptual, they don't try to do too much, and they don't intimidate the visitor.

Few cognitive gains emerged from a visit to either of the observatories. This seeming lack of significant learning outcomes is in part due to the fact that serious education was not a primary mandate of each facility, in part due to the fact that most visitors did not arrive expecting or necessarily wanting to learn something, in part symptomatic of less than ideal design strategies, and especially due to a lack of knowledge of visitor demographics. If serious learning objectives are on the agenda then careful consideration must be given not just to the content and presentation of exhibits, but more importantly, to how the content will be received by the typical visitor. Again, this requires visitor studies.

On the other side of the coin, both visitor centres were very successful at producing a strong and lasting affective impact. Visitors' strongest memories were not of the content of the exhibitions, but rather, were experiential, relating strongly to the physical environment-ironically, and perhaps tellingly, that aspect of the visitor centre not controlled by the exhibitions' designers. The information in the centres was incidental to the visit and in the end visitors learned the 'where' and, to a lesser extent, the 'how' of astronomy, rather than the 'what' contained in exhibits. If promoting positive attitudes towards astronomy is the main objective of the visitor centre, then its on-site location is the most important factor. Both sites were successful at achieving this outcome.

An important lesson emerges from this discussion. Clearly it is not that the information in the visitor centres did not matter to visitors-indeed, most visitors spent a great deal of time carefully reading text and using exhibits. Furthermore, most visitors themselves perceived their visit as being a learning experience, even if it could not be substantiated by interviews. What the onsite visitor centre provided for the casual visitor, and what the casual visitor valued most, was the opportunity to visit, and to see the instruments of exploration firsthand and close up. This access gave visitors a sense of ownership and connection with the scientific community, and with the universe in which they live. However, though access and location had more of an impact on visitors than exhibition material, this is not a license to put less than carefully scrutinised material within the facility. 
Overall, the visitor centres in this study were found to be very effective at producing an affective response, and less effective at producing cognitive gains that could be characterised as a true learning experience. Whether these were the intentions of the observatories is not entirely clear, which is part of the problem. Clearly defined detailed goals are necessary to designing and constructing effective exhibits. Most importantly, knowledge of the visitor (interests, dislikes, misconceptions, visit motivations) is crucial to designing exhibits that will communicate and have the potential to truly educate.

\section{References}

Burtnyk, K. 2000, On-site insights: Visitor impressions of astronomy exhibits at observatory visitor centres. Master's thesis. Australian National University, Canberra

Falk, J. H., \& Dierking, L. D. 1992, The Museum Experience (Washington: Whalesback Books)

Perry, D. L. 1993, Beyond cognition and affect: The anatomy of a museum visit. Proc. Conf. on Visitors Studies, ed. D. Thompson et al., Visitor Studies Association, Albuquerque, NM, Jacksonville, AL, pp. 43-7

Rennie, L., \& McClafferty, T. 1996, 'Science centres and science learning' in Studies in Science Education, Vol. 27, ed. E. Jenkins \& J. Donnelly, Centre for Studies in Science and Mathematics Education, University of Leeds, pp. 53-98 